\title{
PSEUDOTUMOR INFLAMATORIO VESICAL
}

\author{
J.M. ABASCAL JUNQUERA, E. TRILLA HERRERA, S. ESQUENA FERNÁNDEZ, \\ C. RAMÍREZ SEVILLA, R. MARTOS CALVO, F. SERRALLACH OREJAS, \\ Y. ID M HÁMMED, I. DE TORRES*, J. MOROTE ROBLES
}

Servicio de Urología. *Servicio de Anatomía Patológica. Hospital General Universitario Vall d'Hebron. Barcelona.

Actas Urol Esp. 28 (1): 62-64, 2004

\section{RESUMEN}

PSEUDOTUMOR INFLAMATORIO VESICAL

OBJETIVO: Presentar un nuevo caso de pseudotumor inflamatorio vesical.

MATERIAL Y MÉTODOS: Se describe la clínica, diagnóstico y tratamiento de un caso de pseudotumor inflamatorio de localización vesical en un varón de 22 años sin antecedentes urológicos de interés.

CONCLUSIONES: El pseudotumor inflamatorio es una lesión benigna poco frecuente y de etiología desconocida. El principal diagnóstico diferencial morfológico es el rabdomiosarcoma vesical. El estudio inmunohistoquímico es fundamental en el diagnóstico, caracterización y diferenciación de ambas patologias. Su carácter benigno obliga a un tratamiento conservador siempre que sea posible.

PALABRAS CLAVE: Pseudotumor inflamatorio. Hematuria.

\section{ABSTRACT \\ INFLAMMATORY PSEUDOTUMOR OF THE URINARY BLADDER}

OBJECTIVE: To present a new case of inflammatory pseudotumor of the urinary bladder.

MATERIAL AND METHODS: We describe the clinical, diagnostic and treatment of a new case of inflammatory pseudotumor of the urinary bladder in a 22 years old man without urological history.

CONCLUSIONS: The inflammatory pseudotumor of the bladder is a rare benign lesion of unknown etiology. The most important histopathological differential diagnosis to consider is the rabdomyosarcoma. The immunohistochemistry study is basic in the diagnostic, characterization and differentiation of both pathologies. His benign developpement force us to a conservative management always it is possible.

KEY WORDS: Inflammatory pseudotumor. Hematuria.

L a mayoría de las neoplasias vesicales proceden del epitelio transicional. Con menor frecuencia se originan del componente mesodérmico, donde se incluyen diferentes lesiones cuyo representante más común es el leiomioma benigno.

El pseudotumor inflamatorio de la vejiga fue descrito por primera vez en el año 1985 y desde entonces, y dado su carácter excepcional, existen pocos casos descritos en la literatura médica. A pesar de considerarse una lesión de naturaleza benigna, representa un reto en el diagnóstico histológico dada su aparente similitud con el rabdomiosarcoma vesical. Los estudios inmunohistoquímicos permiten su diferenciación y, por tanto, el diagnóstico correcto.

A continuación presentamos un nuevo caso de pseudotumor inflamatorio de localización vesical, analizando sus características clínicas, diagnósticas y anatomopatológicas. 


\section{CASO CLÍNICO}

Varón de 22 años de edad sin hábitos tóxicos ni alergias medicamentosas conocidas. Sin antecedentes familiares de interés. Consulta por cuadro de hematuria macroscópica con coágulos de varios días de evolución. El paciente no refiere fiebre, clínica lumbar ni miccional. No antecedentes traumáticos. A la exploración el paciente se encuentra afebril, con cierta palidez mucocutánea. El abdomen es blando, depresible, sin masas palpables. El tacto rectal demuestra una próstata pequeña, elástica, con sensación de ocupación a nivel supraprostático. La analítica sanguínea y la radiografía simple de abdomen resultaron anodinas. En la ecografía renovesicoprostática, se objetivó una masa heterogénea e hipervascularizada de $7 \times 7 \mathrm{~cm}$ dependiente de la pared posterosuperior de la vejiga (Fig. 1). Dado el carácter sangrante de la lesión, se decide la práctica de una resección transuretral, apreciándose una tumoración bien delimitada en cara posterior vesical con ulceración central. Se practica resección parcial. Asímismo, se practicó estudio de extensión mediante tomografía axial computerizada (TAC), que informó de lesión tumoral intra-

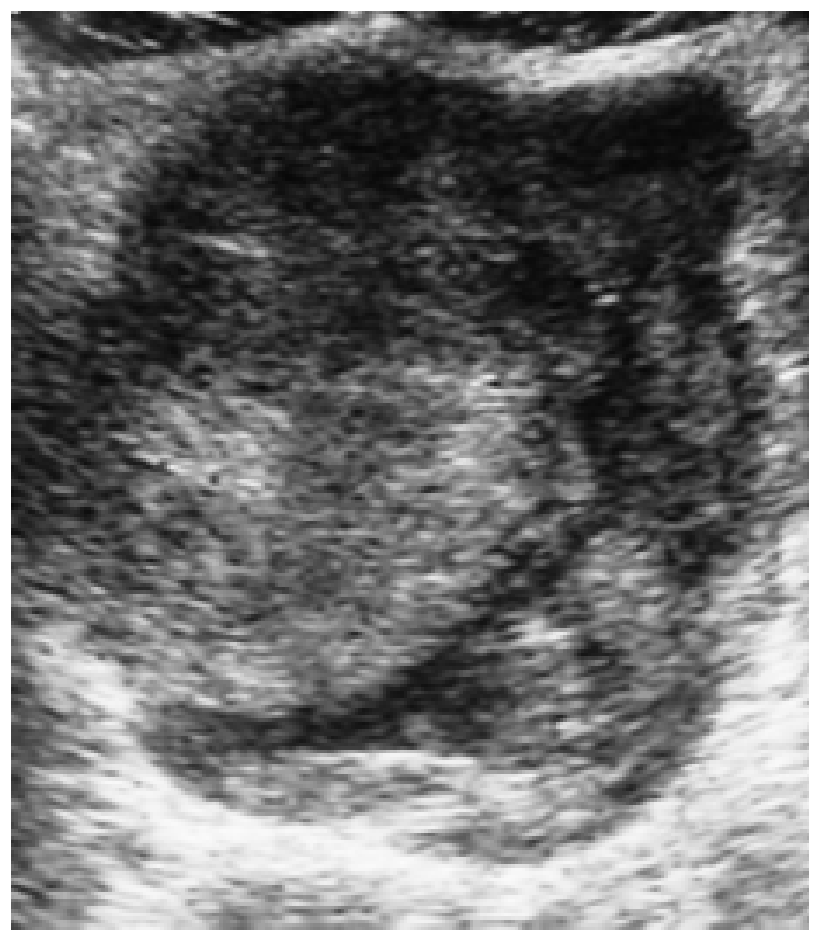

FIGURA 1. Ecografia vesical: gran masa heterogénea dependiente de pared posterosuperior derecha. vesical sin evidencia de extensión locorregional ni a distancia (Fig. 2). El informe anatomopatológico informó de pseudotumor inflamatorio de vejiga (tumor pseudosarcomatoso miofibroblástico). Tras la resección endoscópica y dado el carácter benigno de la lesión, se decidió la realización de cirugía conservadora vesical, practicándose cistectomía parcial (Fig. 3). El estudio histológico e inmunohistoquímico corroboró el diagnóstico de pseudotumor inflamatorio. Actualmente, pasados seis meses de la intervención el paciente se encuentra asintomático y sin evidencias radiológicas de recidiva.

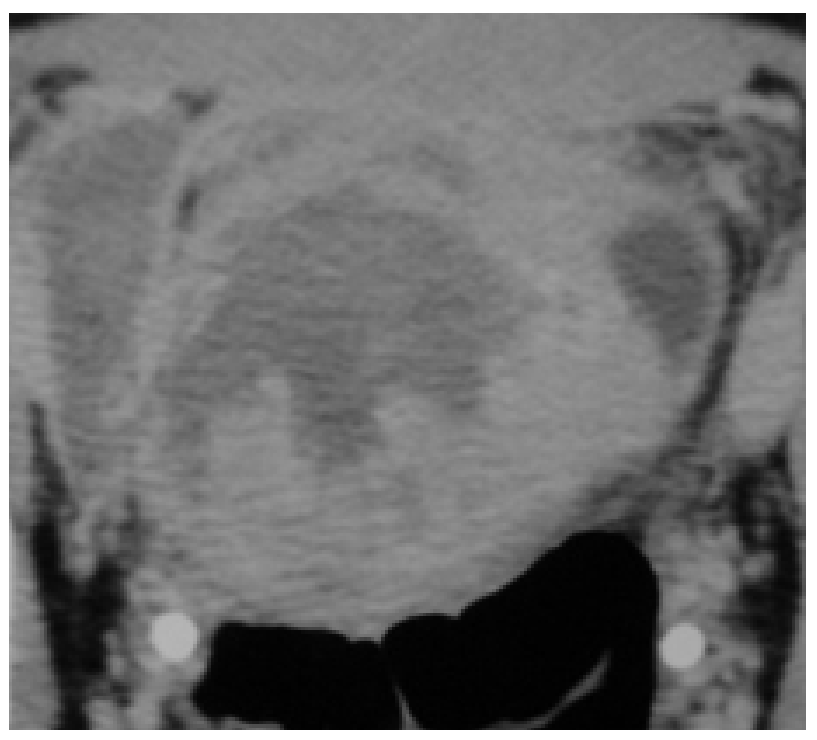

FIGURA 2. TAC abdomino-pélvico: engrosamiento difuso de las paredes vesicales. Uréteres en su tramo distal no dilatados.

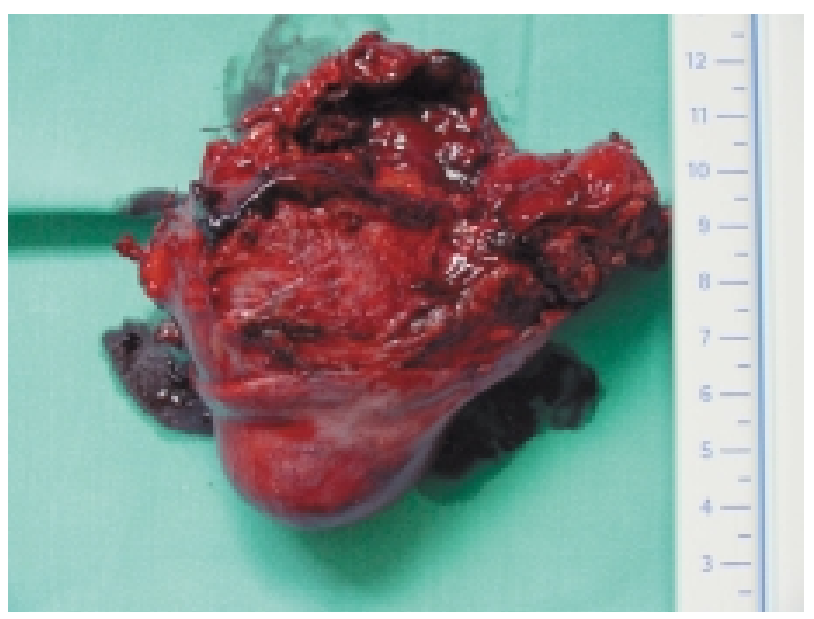

FIGURA 3. Pieza macroscópica tras la cistectomia parcial. 


\section{DISCUSIÓN}

Entre el 1 y el 5\% de todos los tumores vesicales tienen un origen no transicional. En su mayoría se trata de leiomiomas o, en caso de tumoraciones malignas, leiomiosarcomas infiltrantes ${ }^{1}$. Ocasionalmente se trata de lesiones de carácter pseudoinflamatorio de difícil diferenciación histopatológica. En 1985 Orenstein y cols. describen por primera vez dos pacientes que presentaban aparentemente un sarcoma vesical pero que el estudio histopatológico completo posterior reveló una fascitis nodular a nivel vesical, entidad considerada hasta el momento como una tumoración mesenquimatosa benigna generalmente localizada en las extremidades ${ }^{2}$.

Existe una gran variedad de términos con los que se ha designado a este tipo de lesiones: pseudosarcoma reactivo, nódulo post-operatorio de células en huso, pseudotumor inflamatorio, tumor fibromatoso pseudosarcomatoso, proliferación miofibroblástica pseudosarcomatosa, etc. $^{3}$. Esta variedad de denominaciones responde a la heterogeneidad histológica de estas lesiones; aunque siempre predominan las células fibroblásticas y mesenquimales de morfología fusiforme, otros tienen un predominio eosinofílico, etc. ${ }^{4}$.

El origen de este tipo de lesiones inflamatorias es desconocido, algunos autores creen que se trataría de lesiones tisulares reactivas post-operatorias $^{5}$; en otras ocasiones se asocian a factores ambientales como el tabaco o exposición a otros agentes carcinógenos ${ }^{6}$. Así mismo, se han implicado determinadas alteraciones metabólicas o la presencia de patología vesical previa como la cistitis eosinofílica ${ }^{7}$. En la mayoría de las ocasiones no se identifican factores predisponentes.

La hematuria macroscópica es la forma de inicio más habitual, si bien se han descrito otros sintomas menos frecuentes como dolor abdominal inespecífico y síntomas del tracto urinario inferior de carácter crónico.

Histológicamente se caracteriza por ser una tumoración compuesta de células elongadas en forma de huso (células fusiformes) con citoplasma eosinofilico. Las mitosis son poco frecuentes y cuando aparecen lo hacen de manera típica. El estroma tumoral que rodea a las células suele ser de naturaleza mixoide, rico en mucopolisacáridos. Suelen presentar una rica vascularización lo que favorece el sangrado. Es característico el hallazgo de un infiltrado inflamatorio crónico de células mononucleares ${ }^{8}$.

El diagnóstico diferencial hay que plantearlo con el rabdomiosarcoma vesical primario, morfológicamente indiferenciable, si bien el estudio inmunohistoquímico completo permitirá la diferenciación de ambos procesos (negatividad de las inmunotinciones para desmina y miogenina a diferencia del rabdomiosarcoma). Ante la mínima duda, es recomendable un estudio de extensión de la enfermedad (tomografía axial computerizada toraco-abdominal y gammagrafía ósea).

Dado el carácter benigno del pseudotumor inflamatorio vesical, el tratamiento conservador (resección endoscópica y/o cistectomía parcial), siempre que sea posible, debería ser la norma. A pesar del buen pronóstico inicial (no hay descritos casos de enfermedad a distancia en la literatura publicada), es recomendable un seguimiento estricto de estos pacientes.

\section{REFERENCIAS}

1. NOCHOMOVITZ L, ORENSTEIN J.: Inflammatory pseudotumor of the urinary bladder-possible relationship to nodular fascitis. Two cases reports, cytologic observations, and ultraestructural observations. Amer J Surg Path 1985; 9: 366-369.

2. RO J, AYALA A, ORDÓÑEZ N, SWANSON D, BABAIAN R.: Pseudosarcomatous fibromyxoid tumor of the urinary bladder. Amer J Clin Path 1986; 86: 583-585.

3. STARK G, FEDDERSEN R, LOWE B, BENSON C, BLACK W, BORDEN T.: Inflammatory pseudotumor (pseudosarcoma) of the bladder. J Urol 1989; 141: 610-613.

4. DIETRICK D, KABALIN J, DANIELS G, EPSTEIN A, FIELDING I.: Inflammatory pseudotumor of the bladder. J Urol 1992; 148: 141-144.

5. DAS S, UPTON J, AMAR A.: Nodular fascitis of the bladder. J Urol 1988; 140: 1532-1534.

6. ROTH J.: Reactive pseudosarcomatous response in urinary bladder. Urology 1980; 16: 635-636.

7. PROPPE K, SCULLY R, ROSAI J.: Postoperative spindle cell nodules of genitourinary tract resembling sarcomas. A report of eight cases. Amer J Surg Path 1984; 8: 101-102.

8. HUANG W, RO J, GRIGNON D, SWANSON D, ORDÓÑEZ N, AYALA A.: Postoperative spindle cell nodule of the prostate and bladder. J Urol 1990; 143: $824-826$.

Dr. J.M. Abascal Junquera

Servicio de Urología. Hospital Vall d'Hebrón

Passeig Vall d'Hebrón, 119-129

08035 Barcelona

(Trabajo recibido el 29 julio de 2003) 